\title{
Intraoperative Radiation Therapy: A Critical Analysis of the ELIOT and TARGIT Trials. Part 2-TARGIT
}

\author{
Melvin J. Silverstein, $\mathrm{MD}^{1,2}$, Gerd Fastner, $\mathrm{MD}^{3}$, Sergio Maluta, $\mathrm{MD}^{4}$, Roland Reitsamer, $\mathrm{MD}^{5}$, Donald A. Goer, \\ $\mathrm{PhD}^{6}$, Frank Vicini, $\mathrm{MD}^{7}$, and David Wazer, $\mathrm{MD}^{8,9}$ \\ ${ }^{1}$ Breast Center, Hoag Memorial Hospital Presbyterian, Newport Beach, CA; ${ }^{2}$ Department of Surgery, Keck School of \\ Medicine, University of Southern California, Los Angeles, CA; ${ }^{3}$ Radiotherapy and Radio-Oncology Landeskrankenhaus, \\ Paracelsus Medical University, Salzburg, Austria; ${ }^{4}$ Radiation Oncology, Oncological Hyperthermia Unit—Medical Center, \\ Verona, Italy; ${ }^{5}$ Department of Senology, Paracelsus Medical University Salzburg, Salzburg, Austria; ${ }^{6}$ Physics, IntraOp \\ Medical, Sunnyvale, CA; ${ }^{7}$ Radiation Oncology, St. Joseph Mercy Oakland, Pontiac, MI; ${ }^{8}$ Radiation Oncology, Tufts \\ University School of Medicine, Boston, MA; ${ }^{9}$ Radiation Oncology, Albert Medical School of Brown University, \\ Providence, RI
}

\begin{abstract}
Background. Two randomized intraoperative radiation therapy trials for early-stage breast cancer were recently published. The ELIOT Trial used electrons (IOERT), and the TARGIT-A Trial Update used 50-kV X-rays (IORT). These studies were compared for similarities and differences. The results were analyzed and used to determine which patients might be suitable for single-dose treatment. Methods. The primary sources of data were the ELIOT Trial and TARGIT-A Trial, as well as a comprehensive analysis of the peer-reviewed literature of accelerated partial breast irradiation (APBI) using 50-kV X-rays or electrons. Studies published or presented prior to March 2014 were analyzed for efficacy, patient restrictions, complications, and outcome.

Results. With a median follow-up of 5.8 years, the 5-year recurrence rates for ELIOT versus EBRT patients were 4.4 and $0.4 \%$, respectively, $p=0.0001$. A low-risk ELIOT group was identified with a 5-year recurrence rate of $1.5 \%$. With a median follow-up of 29 months, the 5-year recurrence rates for the TARGIT-A versus EBRT patients were 3.3 and $1.3 \%$, respectively, $p=0.042$.

Conclusions. With 5.8 years of median follow-up, IOERT appears to have a subset of low risk women for whom IOERT is acceptable. With 29 months of median follow-up
\end{abstract}

(C) The Author(s) 2014. This article is published with open access at Springerlink.com

First Received: 23 June 2014;

Published Online: 20 August 2014

M. J. Silverstein, MD

e-mail: melsilver9@gmail.com the results of IORT with 50-kV devices are promising, but longer follow-up data are required. At the current time, single-fraction IOERT or IORT patients should be treated under strict institutional protocols.

In the preceding report (Part 1) in this issue of the Annals, we outline the rationale for intraoperative radiation therapy (IORT) and begin a critical analysis of the 2 prospective randomized trials currently published. Part 1 discusses the ELIOT Trial, a trial using electrons during surgery as the entire radiation therapy treatment. In this report, we continue with a critical analysis of the TARGITA Trial, a trial that used $50-\mathrm{kV}$ x-rays rather than electrons.

\section{METHODS}

See Part I for methods used in the analysis.

\section{TARGIT-A Trial}

Overview The TARGIT-A Trial randomized 3,451 patients either to standard EBRT treatment or to TARGIT-A. Eligibility criteria were age $\geq 45$ years, tumor size $\leq 3.5 \mathrm{~cm}$, N0-1, M0, and unifocal invasive ductal carcinoma. If the participating institution determined the patient was at high risk for recurrence, an additional 5 weeks of EBRT was given, calling this "riskadapted IORT." The Trial began in March 2000. Beginning in 2004, approximately $30 \%$ of the patients had TARGIT-A after final pathology in a second surgical 


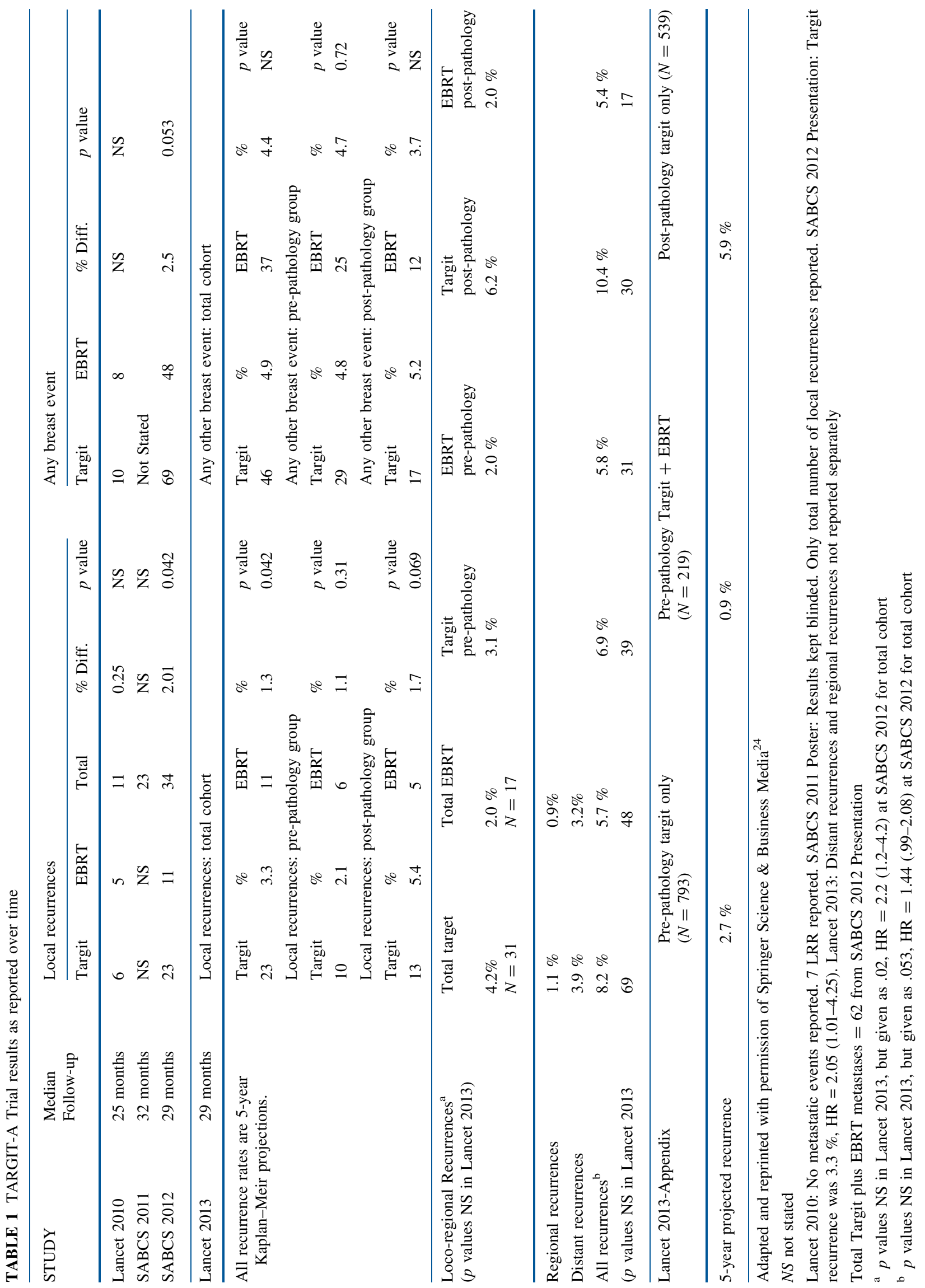


Ipsilateral Breast Recurrence

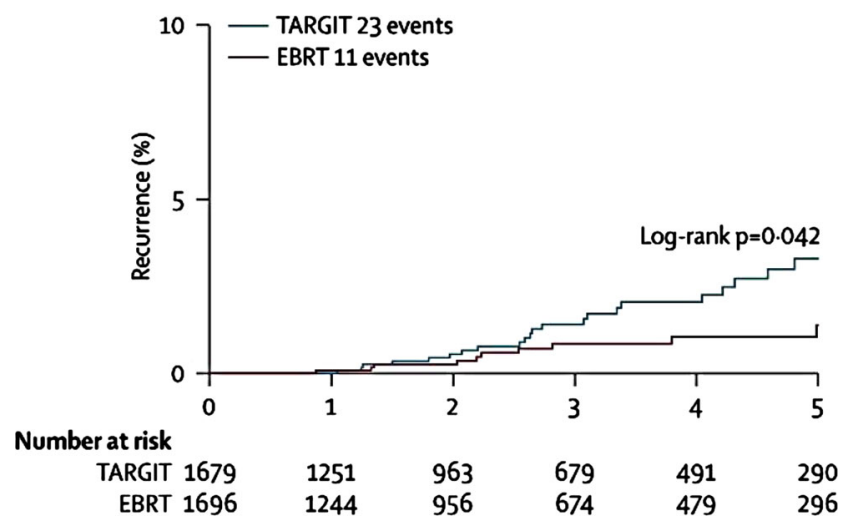

(a) IBTR, 3.3\% Targit, 1.3\% EBRT, p=0.042

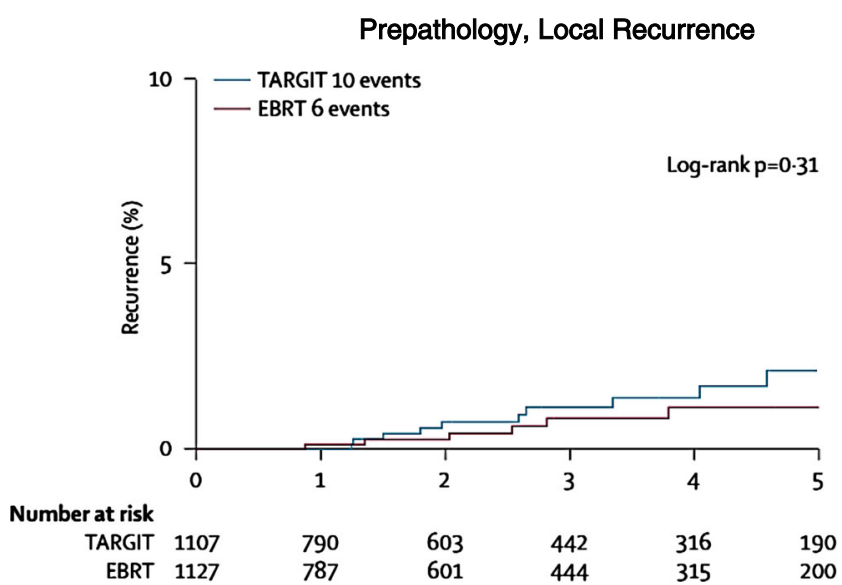

(c) IBTR, pre-pathology

$2.1 \%$ Targit, $1.1 \%$ EBRT, $p=0.31$

*Adapted from Figures 2 and 3 in Lancet ${ }^{7}$

FIG. 1 5-year Kaplan-Meier projections for recurrences from TARGIT-A treated patients vs EBRT treated patients. a Ipsilateral breast recurrence. b Overall breast recurrence. c Prepathology, local

procedure about 30 days after the original surgery. This group was designated the "postpathology" group as opposed to the "prepathology" group who received TARGIT-A during initial tumor surgery. The results for these different patient cohorts are shown in Table 1.

Technique In the prepathology TARGIT-A patients, following tumor excision, an appropriately sized spherical applicator was placed in the tumor bed. Purse string sutures were used to approximate breast tissue at risk to the applicator. Radiation was delivered over $20-45 \mathrm{~min}$

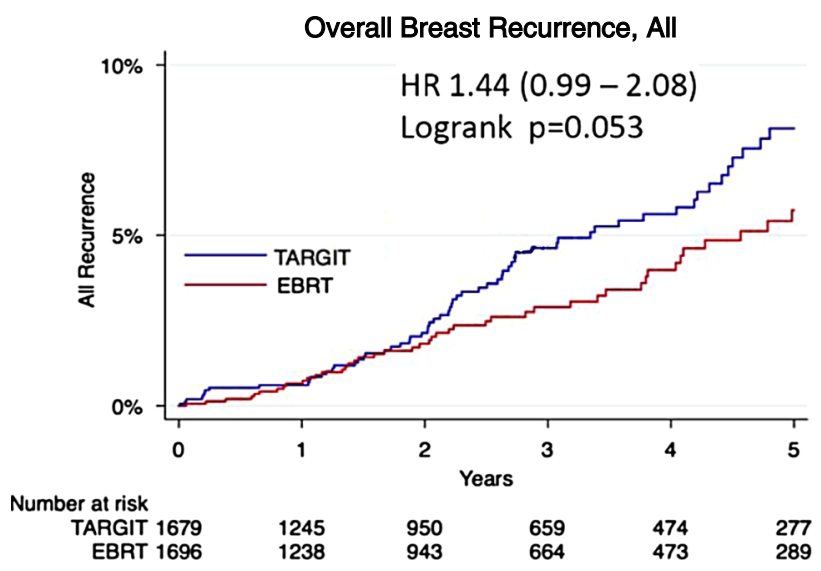

(b) Overall breast cancer recurrence, $\mathrm{p}=0.053$ (lipsilateral, contralateral, axilla and distant) Presented at SABCS, but not in Lancet 2013

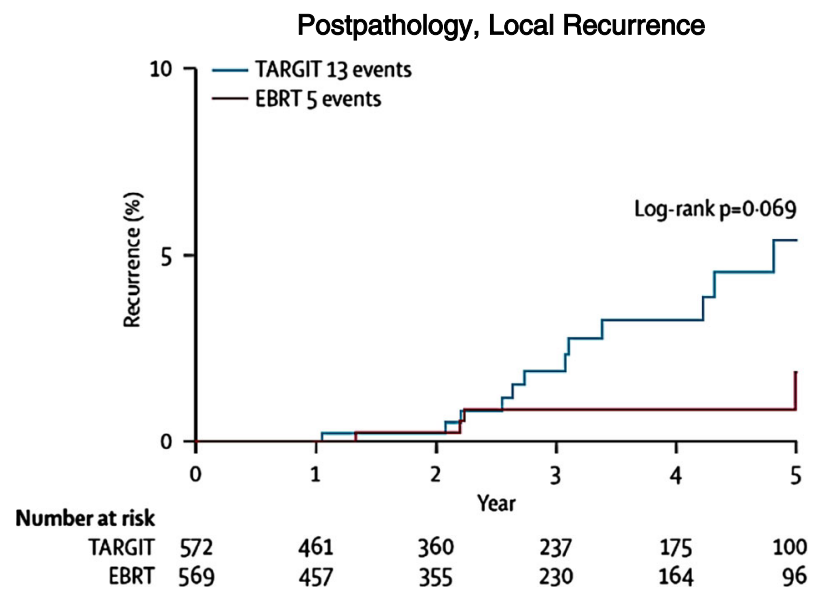

(d) IBTR, post-pathology

5.4\% Targit, $1.7 \%$ EBRT, $p=0.069$

recurrence. d Postpathology, local recurrence. Adapted from Figs. 2 and 3 in Lancet $^{7}$

to the tumor bed, which received $20 \mathrm{~Gy}$ at the surface of the applicator and attenuated to 5-7 Gy at $1-\mathrm{cm}$ depth. If risk factors were found at the time of surgery or postoperatively, when final pathology was available, the 20 Gy TARGIT treatment was considered as a boost, and patients received an additional 50 Gy equivalent of EBRT, delivered over 3-5 weeks, depending on the institutional preference. Institutions were free to determine what risk factors required additional EBRT.

The postpathology TARGIT-A patients received 20 Gy irradiation after final pathology determined no risk 
factors, typically within 30 days of surgical tumor removal.

The EBRT patients, whether prepathology or postpathology, received $3-5$ weeks of $50 \mathrm{~Gy}$ equivalent EBRT \pm boost depending on the institutional preference.

Complications Wound complications were similar between groups, but grade 3 or 4 skin complications were significantly reduced with TARGIT (4 of 1720) vs EBRT (13 of 1731), $p=0.029$.

Regional Failures Regional failures were similar in both groups (8 events for TARGIT vs 6 events for EBRT) $(p=0.6)$.

Results At 29 months of median follow-up, the 5-year risk of local recurrence was $1.3 \%$ for EBRT and $3.3 \%$ for all TARGIT-A patients $(p=0.042)$. Target A prepathology patients had a 5-year risk of $2.1 \%$. Postpathology patients had a 5-year risk of $5.7 \%$.

Overall recurrence (ipsilateral breast, contralateral breast, axilla, and distant) showed a worsening trend for TARGIT A compared with EBRT: 69 events vs 48 events $(p=0.053)$. Both postpathology and prepathology TARGIT-A patients had more local recurrences than the EBRT patients, although the difference was not statistically significant. Postpathology patients exceeded the Trial's preset noninferiority margin of $2.5 \%$ (5.4 vs $1.7 \%, p=0.069$ ); prepathology patients did not (2.1 vs $1.0 \%, p=0.31)$. Approximately $21 \%$ of prepathology patients who received TARGIT-A also had 5 weeks of EBRT because of risk factors determined at the time of surgery or when final histopathology was available. Patients who received only TARGIT-A had 3 times the recurrence rate of those who received TARGIT-A plus 5 weeks of EBRT (2.7 vs $0.9 \%$ ). This difference was not significant, but no $p$ value was provided. Ipsilateral breast recurrence rates for all patients, for prepathology and postpathology patients, and for any breast recurrence are shown in Fig. 1.

Survival Breast cancer mortality was similar for TARGIT $(2.6 \%)$ vs EBRT $(1.9 \%), p=0.56$. TARGIT resulted in significantly fewer non-breast-cancer deaths $1.4 \%(n=17)$ vs $3.5 \%(n=35), p=0.0086$. This was due to fewer deaths from cardiovascular causes and other cancers. Overall mortality was $3.9 \%$ for TARGIT versus $5.3 \%$ for EBRT, $p=0.099$.

\section{DISCUSSION}

Between March 2000 and April 2010 2,232 patients were accrued, sufficient for proof of noninferiority. ${ }^{1}$ Results were reported 3 months after completion of accrual when the
TABLE 2 TARGIT-A local recurrence summary by treatment cohort

\begin{tabular}{llll}
\hline Cohort & $\begin{array}{l}\text { Number of } \\
\text { recurrences }\end{array}$ & Percent & $p$ value \\
\hline Prepathology Targit & 10 & $2.1 \%$ & 0.31 \\
Prepathology EBRT & 6 & $1.1 \%$ & \\
Postpathology Targit & 13 & $5.4 \%$ & 0.069 \\
Postpathology EBRT & 5 & $1.7 \%$ & \\
Prepathology Targit alone & $\sim 7^{\mathrm{a}}$ & $2.7 \%$ & Not \\
$\quad(N=793)$ & & & stated \\
Prepathology Targit + boost & $\sim 3^{\mathrm{a}}$ & $0.9 \%$ & \\
$\quad(N=219)$ & & & \\
\hline
\end{tabular}

Reprinted with permission of Springer Science \& Business Media ${ }^{24}$

${ }^{a}$ Number of recurrences extrapolated from presented data

median follow-up was 25 months. ${ }^{1}$ The authors maintained early publication was justified because proof of noninferiority required only 585 patients, and they had reached that number with a 4.6-year median follow-up. Also they said peak recurrences for breast cancer occur in years 2 and 3, offering in support that no recurrences were seen in year 4 . At that time, critics expressed concern mainly about the immaturity of the data. ${ }^{2-6}$ Accrual and randomization of 1,219 additional patients continued until June 2012, increasing the Trial population to 3,451 patients, resulting in a median follow-up of just 29 months. ${ }^{7}$

The TARGIT-A update shows recurrences in both the TARGIT and EBRT groups in year 4 .

At the time of the update, the 5 EBRT recurrences initially reported more than doubled to 11 , and the six initial TARGIT recurrences had almost quadrupled to 23 , questioning the claim of a recurrence peak at 2 or 3 years. ${ }^{1,7}$ The results of the TARGIT-A trial, with a median followup (FU) of 29 months, is still well below the median time when breast recurrences can be expected, especially since more than $90 \%$ of TARGIT-A women were estrogen receptor positive, and at least $65 \%$ received adjuvant hormonal therapy, a treatment well-known to delay recurrences in ER + women. ${ }^{1,7-9}$

The authors used binomial proportion statistics to show equivalence between the mature cohort (2,232 patients, median $\mathrm{FU}=3$ years, 7 months), the earliest cohort $(1,222$ patients, median $\mathrm{FU}=5$ years $)$, and the total cohort ( 3,451 patients, median $\mathrm{FU}=2$ years 5 months). Haviland points out that binomial proportion statistics is invalid for follow-ups less than 5 years and that the appropriate statistical methodology is survival analysis for local recurrence. ${ }^{10}$ Only $18 \%$ of patients had a FU of 5 years in the TARGIT-A update. ${ }^{7}$ Haviland estimates the hazard ratio for the reported local recurrence rates and calculates the local recurrence rate for TARGIT-A could be as high as 
$7.1 \%$, far exceeding the noninferiority margin of $2.5 \%$ established by the trial.

The initial TARGIT-A publication did not differentiate between prepathology and postpathology patients or Targit boost patients. ${ }^{1}$ The TARGIT update shows these strata are not equivalent, with postpathology having higher local recurrence rates than prepathology (Table 2), despite postpathology patients presumably being lower risk as the treatment was delivered in a second operation after final pathology. ${ }^{7}$ The authors attribute the difference either to delay in wound fluid suppression of tumor cells, since there is a delay of radiation in postpathology TARGIT, or to a geometric miss when inserting the applicator postsurgery. While geometric miss might partially explain the results, it is not the likely a major cause of their findings. The IORT Intrabeam boost study of 299 patients reported no difference in recurrence rates between prepathology and postpathology patients. ${ }^{11}$ The 5-year recurrence rate for all patients was $1.73 \%$. The authors do not report the median applicator size used in the prepathology and postpathology patients, but if the median sizes reported in other Intrabeam publications are used, it is likely that postpathology patients had irradiated tissue volumes less than half the volumes in prepathology patients. ${ }^{11,12}$ In IORT boost, EBRT can compensate for the smaller volume irradiated in the postpathology patients. One can also see this trend in the prepathology TARGIT patients since TARGIT plus EBRT has three times fewer local recurrences than TARGIT alone even though those who also received 5 weeks of EBRT were presumably at higher risk (Table 2).

The authors note that the difference in IBTR for all patients is still within their absolute noninferiority margin of $2.5 \%$ (Fig. 1a). ${ }^{7}$ Cuzick cautions that the authors have misused the noninferiority criterion, which requires the upper confidence interval (CI) be less than the predefined noninferiority level of $2.5 \% .{ }^{13}$ In the TARGIT-A update, the upper CI was $5.1 \%$, throwing doubt on their assertion of noninferiority. ${ }^{7}$ Looking at the divergence of slopes in Fig. 1a, it appears likely that the $2.5 \%$ noninferiority criterion for IBTR will be exceeded irrespective of the CI upper limit.

Overall breast recurrence rates in the TARGIT group also exceeded rates in the EBRT group (Fig. 1b), a difference at borderline statistical significance $(p=0.053){ }^{14}$ While the difference in breast cancer deaths with TARGIT vs EBRT is not significant (20 deaths, $2.6 \%$ vs. 16 deaths, $1.9 \%, p=0.56$ ), these higher recurrence rates with short follow-up suggests more follow-up is needed.

Follow-up may also be too short to determine whether prepathology TARGIT patients will ultimately do better than the entire TARGIT cohort. The difference between this favorable TARGIT cohort and the EBRT group is $1.0 \%$, with a median follow-up of 29 months, compared with a difference of $.25 \%$ between the TARGIT group and the EBRT group in the initial publication. ${ }^{1,7}$

The TARGIT study involved 33 centers in 11 countries and lasted more than 12 years. A large multi-institutional study such as TARGIT-A demands a high level of control and standardization. However, in TARGIT-A, each center treated the EBRT group according to its own institutional guidelines and could determine its own criteria for which patients would receive TARGIT boost rather than TARGIT APBI.

Sperk et al. analyzed recurrences in the Mannheim cohort of TARGIT-A patients. ${ }^{15}$ Among 54 TARGIT-A patients, $37 \%$ were converted from TARGIT APBI to TARGIT Boost because of risk factors Sperk et al. chose for conversion, which included larger tumors $(>2 \mathrm{~cm})$ with narrower margins $(<10 \mathrm{~mm})$. With a median follow-up of 40 months, they report no recurrences in the 34 patients who received TARGIT APBI. Notably, $80 \%$ of their patients also received adjuvant endocrine therapy, which could delay the appearance of recurrences. Nevertheless, if these good results are sustained with longer follow-up and can be replicated by other centers, it is possible that $\mathrm{T} 1$ tumors and wide excision surgery with adjuvant endocrine therapy could form a basis for "risk-adapted" TARGIT treatment. The variability of standards from center to center in the TARGIT-A Trial makes it more difficult to identify which cohort of women might benefit from this treatment strategy.

Prepathology women meeting the general TARGIT-A inclusion criteria appear to be the best candidates. However, at least $20 \%$ of women who receive TARGIT treatment will also require 5 weeks of EBRT. Because the TARGIT-A study allowed treatment centers to determine the risk factors that required an additional 5 weeks of treatment, the Trial provides no guidance to new adopters as to when it is appropriate to add additional treatment.

The volume of tissue irradiated with the TARGIT technique is of concern because dose decreases rapidly with distance from the applicator surface. Even assuming favorable radiobiological equivalence, only tissue within a few $\mathrm{mm}$ of the applicator surface receives as much as a 50-Gy EBRT equivalent dose.

In the Milan III Trial, quandrantectomy alone was insufficient to achieve local control in early-stage breast cancer, even though $20 \mathrm{~mm}$ of tissue beyond the tumor was excised in all directions. ${ }^{16}$ At 10 years, local recurrence rates in patients receiving quandrantectomy alone vs those also receiving quadrantectomy plus 5 weeks of EBRT was 23.5 versus $5.8 \%$, respectively, with the difference less in older patients.

A multicenter randomized trial in women older than 55 years compared wide excision surgery alone $(1 \mathrm{~cm}$ clear margins) with wide excision surgery plus 5 weeks of EBRT with an EBRT boost. ${ }^{17}$ Almost all patients received 
TABLE 3 Causes of death as reported in TARGIT-A update

\begin{tabular}{|c|c|c|c|c|c|c|c|c|}
\hline & \multicolumn{2}{|l|}{ All deaths } & \multicolumn{6}{|c|}{ Breast deaths and cardiac deaths, only } \\
\hline & TARGIT & EBRT & TARGIT & EBRT & $\begin{array}{l}\text { Targit } \\
\text { prepath }\end{array}$ & $\begin{array}{l}\text { EBRT } \\
\text { prepath }\end{array}$ & Targit postpath & EBRT postpath \\
\hline Breast cancer & 20 & 16 & $20(2.6 \%)$ & $16(1.9 \%)$ & $17(3.3 \%)$ & $15(2.7 \%)$ & $3(1.2 \%)$ & $1(0.5 \%)$ \\
\hline Other cancers & 8 & 16 & $p=0.56$ & & $p=0.72$ & & $p=0.35$ & \\
\hline Cardiac death & 2 & 8 & 2 & 8 & NS & NS & NS & NS \\
\hline Strokes & 0 & 2 & & & & & & \\
\hline Ischemic bowel & 0 & 1 & & & & & & \\
\hline Other deaths & 7 & 8 & & & & & & \\
\hline Total & 37 & 51 & $22^{\mathrm{a}}$ & $24^{\mathrm{a}}$ & & & & \\
\hline
\end{tabular}

Adapted from Table 2, Lancet $^{7}$ w/Breast Cancer Deaths added

NS not stated

${ }^{a}$ Death due to breast cancer and cardiac events together

adjuvant hormonal therapy. With a median follow-up of 9 years, the local recurrence rates were $4.4 \%$ for excision alone versus $3.4 \%$ for excision plus radiation therapy, $p=\mathrm{NS}$.

In TARGIT-A, the combination of surgical excision and effective radiation treatment depth is less than in Milan III, and in some cases, even less than $10 \mathrm{~mm}$ total. At 29 months median follow-up, the TARGIT-A postpathology (all of whom received a single-dose treatment in a second procedure) had local recurrence rates of $5.7 \%$, whereas prepathology patients $(21 \%$ of whom also received 5 weeks of WBI) had local recurrence rates of $2.1 \%$.

Fewer deaths were observed in the TARGIT arm than the EBRT arm, 37 versus 51, $p=0.008$ (Table 3). The TARGIT authors assert that TARGIT treatment, while resulting in higher local recurrence rates, leads to an overall improvement in survival due to fewer non-breast cancer deaths. This conclusion is one of the main findings in the TARGIT-A update publication. ${ }^{7}$ The authors recommend that clinicians advise patients that while TARGIT bears a higher risk of local recurrence, TARGIT may decrease overall mortality by $2.3 \%$.

Harness et al. and Yarnold et al. argue that it is impossible for the 12-year-old Targit study, with a median follow-up of 29 months, to impact other cancer deaths, since the latency period for inducing non-breast cancers from breast treatment is known to be 15-20 years. ${ }^{18,19}$ Furthermore, deaths from stroke and ischemic bowel disease cannot be attributed to breast irradiation. If you include only cardiac and breast cancer deaths, the difference between treatment arms is only two patients. Significance in only achieved by including deaths that are unrelated to radiation treatment.
Mackenzie et al., Yarnold et al., and Harness et al. argue that Vaidya et al.'s assertion (fewer cardiac deaths from TARGIT) is inconsistent with the Darby study, the very study cited ${ }^{1}$ in support of this claim. ${ }^{1,718-22}$ Mackenzie et al. suggest differences in baseline cardiac risk factors in the study groups are the most likely explanation for finding more cardiac deaths in the EBRT arm. ${ }^{20}$ Vaidya et al. concede that cardiovascular assessment was not recorded prior to study entry, but speculates that IORT of the tumor bed might have systemic beneficial effects that contribute to reduction in non-breast cancer mortality. ${ }^{22}$ However, this theory was not confirmed in the more mature ELIOT study, which showed no differences in non-breast cancers and overall survival, even out to 10 years of follow-up. ${ }^{23}$

\section{TARGIT-A CONCLUSIONS}

The TARGIT-A trial, like the ELIOT Trial, included patients that today would not be considered the best choice for APBI. TARGIT-A has contributed to our understanding of whether a 1-day treatment may be possible, this time using 50-kV X-rays. With 29 months of median follow-up, the TARGIT Data are still immature and risk-adapted IORT with $50-\mathrm{kV} \mathrm{X}$-rays is still too early in follow-up to select the subset of women whose local control will be within their noninferiority criteria margin of $2.5 \%$. Prepathology patients who meet the TARGIT-A inclusion criteria appear to be the best candidates and, at this point, show encouraging results. Until the data are more mature, $50-\mathrm{kV}$ patients should be treated under strict institutional protocols. When long-term results are available, it is likely there will be a higher overall recurrence rate for TARGIT when compared with EBRT, but, as with ELIOT, we may be able to select subgroups of favorable patients where this 
difference is small and acceptable. How much additional risk of local recurrence is acceptable will vary with patients and the situation in which they find themselves.

OPEN ACCESS This article is distributed under the terms of the Creative Commons Attribution License which permits any use, distribution, and reproduction in any medium, provided the original author(s) and the source are credited.

\section{REFERENCES}

1. Vaidya JS, Joseph DJ, Tobias JS, Bulsara M, Wenz F, Saunders $\mathrm{C}$, et al. Targeted intraoperative radiotherapy versus whole breast radiotherapy for breast cancer (TARGIT-A trial): an international, prospective, randomised, non-inferiority phase 3 trial. Lancet. 2010;376:91-102.

2. Cameron D, Kunkler I, Dixon M, Jack W, Homas J, Kerr G. Intraoperative radiotherapy for early breast cancer. Lancet. 2019;376:1142

3. Haviland J, A'Hern R, Bliss J. Intraoperative radiotherapy for early breast cancer. Lancet. 2010;376:1142-3.

4. Reitsamer R, Fastner G, Kopp M, Menzel C, Sedlmayer F. Intraoperative radiotherapy for early breast cancer. Lancet. 2010;376:1141

5. Sautter-Bihl ML, Sedlmayer F, Budach W, Dunst J, EngenhartCabillic R, Fietkau R, et al. Intraoperative radiotherapy as accelerated partial breast irradiation for early breast cancer : beware of one-stop shops? Strahlenther Onkol. 2010;186:651-7.

6. Smith B, Buchholz T, Kuerer H. Intraoperative radiotherapy for early breast cancer. Lancet. 2010;376:1141.

7. Vaidya JS, Wenz F, Bulsara M, Tobias JS, Joseph DJ, Keshtgar $\mathrm{M}$, et al. Risk-adapted targeted intraoperative radiotherapy versus whole-breast radiotherapy for breast cancer: 5-year results for local control and overall survival from the TARGIT-A randomized trial. Lancet. 2013;383:603-13.

8. Davies C, Pan H, Godwin J, Gray R, Arriagada R, Raina V, et al. Adjuvant Tamoxifen: Longer Against Shorter (ATLAS) Collaborative Group. Long-term effects of continuing adjuvant tamoxifen to 10 years versus stopping at 5 years after diagnosis of oestrogen receptor-positive breast cancer: ATLAS, a randomised trial. Lancet. 2013;381:805-16.

9. Hughes KS, Schnaper LA, Bellon JR, Cirrincione CT, Berry DA, McCormick B, et al. Lumpectomy plus tamoxifen with or without irradiation in women age 70 years or older with early breast cancer: long-term follow-up of CALGB 9343. J Clin Oncol. 2013;31:2382-7.

10. Haviland J, A'Hern R, Bentzen S, Whelan T, Bliss J. Radiotherapy for breast cancer, the TARGIT-A trial. Lancet. 2014;383:1716-7.
11. Vaidya JS, Baum M, Tobias JS, Wenz F, Massarut S, Keshtgar $\mathrm{M}$, et al. Long-term results of targeted intraoperative radiotherapy (targit) boost during breast-conserving surgery. Int $J$ Radiat Oncol Biol Phys. 2011;81:1091-7.

12. Wenz F, Welzel G, Blank E, Hermann B, Steil V, Sütterlin M, et al. Intraoperative radiotherapy as a boost during breast-conserving surgery using low-kilovoltage x-rays: the first 5 years of experience with a novel device. Int J Radiat Oncol Biol Phys. 2010;77:1309-14.

13. Cuzick J. Radiotherapy for breast cancer, the TARGIT-A trial. Lancet. 2014;383:1716.

14. Vaidya JS, Wenz F, Bulsara M, Joseph D, Tobias JS, Kreshtgar $\mathrm{M}$, et al. Targeted intraoperative radiotherapy for early breast cancer: TARGIT-A trial-updated analysis of local recurrence and first analysis of survival. Paper presented at: 35th Annual CTRCAACR San Antonio Breast Cancer Symposium, Dec 4-8, 2012; San Antonio, Texas.

15. Sperk E, Welzel G, Keller A, Kraus-Tiefenbacher U, Gerhardt A, Sütterlin $\mathrm{M}$, et al. Late radiation toxicity after intraoperative radiotherapy (IORT) for breast cancer: results from the randomized phase III trial Targit A. Breast Cancer Res Treat. 2012;135:253-60.

16. Veronesi U, Marubini E, Mariani L, Galimberti V, Luini A, Veronesi $\mathrm{P}$, et al. Radiotherapy after breast-conserving surgery in small breast carcinoma: long-term results of a randomized trial. Ann Oncol. 2001:997-1003.

17. Tinterri C, Gatzemeier W, Costa A, Gentilini MA, Zanini V, Regolo L, et al. Breast-conservative surgery with and without radiotherapy in patients aged 55-75 years with early-stage breast cancer: a prospective, randomized, multicenter trial analysis after 108 months of median follow-up. Ann Surg Oncol. 2014;21:408-15.

18. Harness J, Silverstein M, Wazer D, Riker A. Radiotherapy for breast cancer, the TARGIT-A trial. Lancet. 2014;383:1718-9.

19. Yarnold J, Offersen B, Olivotto I, Poortmans P, Sarin R. Radiotherapy for breast cancer, the TARGIT-A trial. Lancet. 2014;383:1717-8.

20. Mackenzie P, Fyles A, Chung C. Radiotherapy for breast cancer, the TARGIT-A trial. Lancet. 2014;383:1717.

21. Darby S, Ewertz M, Hall P. Ischemic heart disease after breast cancer radiotherapy. N Engl J Med. 2013;368:2527.

22. Vaidya J, Wenz F, Bulsara M, Tobias J, Joseph D, Baum M. Radiotherapy for breast cancer the TARGIT-A trial-Authors' reply. Lancet. 2014;383:1719-20.

23. Veronesi U, Orecchia R, Maisonneuve P, Viale G, Rotmensz N, Sangalli $\mathrm{C}$ et al. Intraoperative radiotherapy versus external radiotherapy for early breast cancer (ELIOT): a randomized controlled equivalence trial. Lancet Oncol. 2013;14:1269-77.

24 Goer D, Silverstein MJ. The emerging role of intraoperative radiation therapy (IORT) in breast cancer. In: Riker A, Krueger B (eds) Breast disease: comprehensive management. New York, Heidelberg, London: Springer Science; 2014. 